\title{
Die Buchstabenwelten von Josua Reichert
}

Bibliotheken sind die Hüter der Worte, die in den Millionen Bänden und hunderten von Millionen Seiten geborgen (oder verborgen?) sind und die ihre Lesesäle und Magazine dem Leser bereithalten. Wie kein anderer Künstler vor und nach ihm hat Josua Reichert Buchstaben und Wörter als eigenständige Elemente der Kunst eingesetzt und mit der unfasslichen Vielfalt seiner Buchstabenund Wortbilder das Urelement alles Schreibens und Druckens geradezu herauspräpariert und ins Bewusstsein gebracht.

"Zweitausend Jahre lang, bevor er die Welt erschuf, betrachtete der Heilige, er sei gesegnet, die Buchstaben und spielte mit ihnen".

In dieser poetischen Vision im kabbalistischen Buch "Schöpfungsalphabet aus dem Buch Sohar", das Josua Reichert 1998 mit einem hebräischen Alphabet veröffentlichte, kann man das ganz den Buchstaben und Wörtern gewidmete Werk dieses Künstlers beschrieben sehen, der ebenso schöpferisch mit den Buchstaben "spielte".

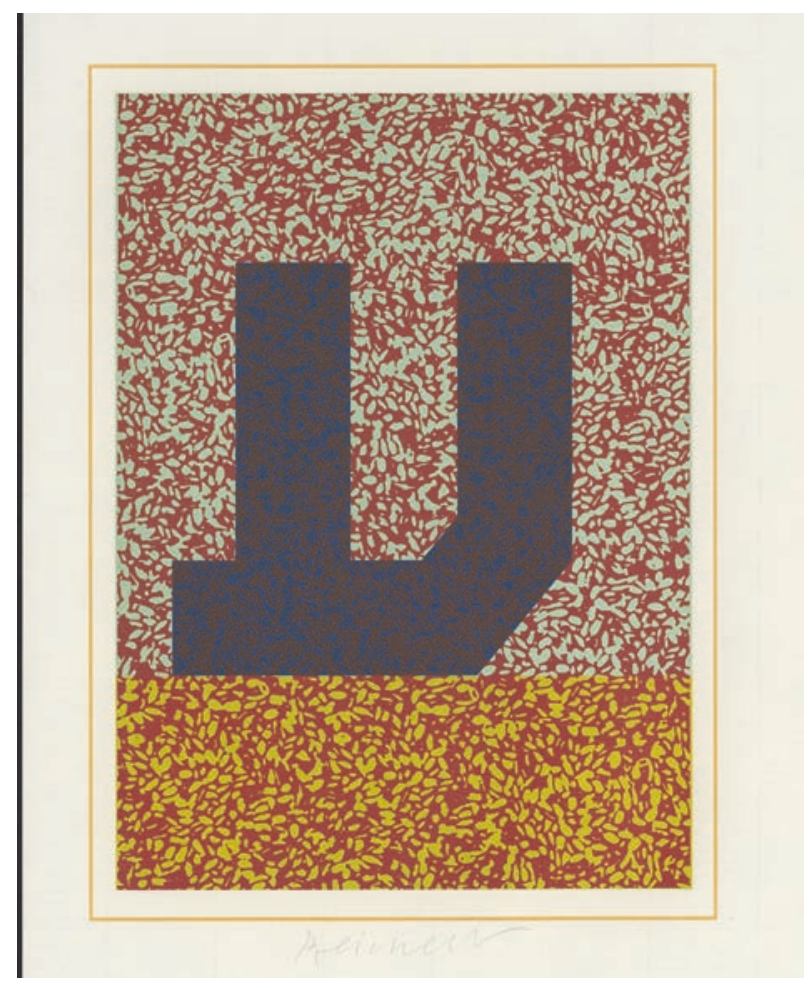

Abb. 1: Hebräischer Buchstabe Ajin aus Josua Reichert: Schöpfungsalphabet. Faber \& Faber, 1998
In seinem Werk verschränken sich Buchstaben als Bildelemente, und werden Blätter zum Anschauen einerseits. Daneben stehen andererseits Drucke, die vorwiegend textbezogen, also zum Lesen sind: Schauen und Lesen machen das Proprium von Reicherts Werk aus. Es war eine sehr glückliche Entscheidung, den noch jungen Künstler Josua Reichert mit der Gestaltung der Räume der Württembergischen Landesbibliothek zu beauftragen. So ist man überall im Gebäude mit einem wegweisenden Hauptwerk konfrontiert - den 36 großformatigen Blättern der sogenannten ,Stuttgarter Drucke'.

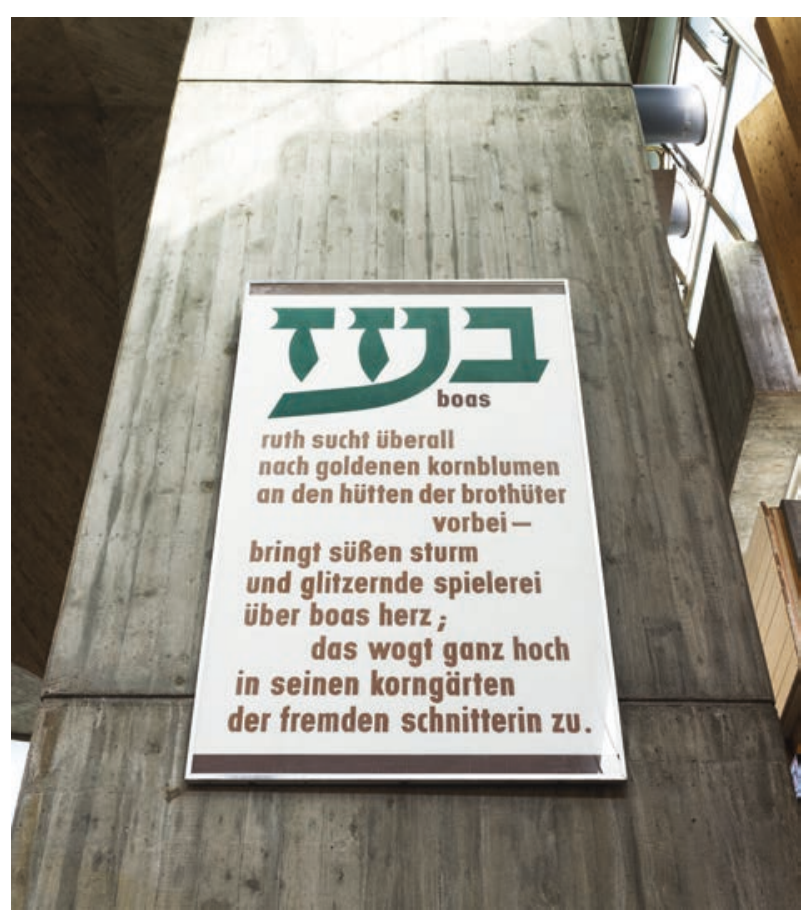

Abb. 2: Stuttgarter Drucke: Boas (Foto: Rafael Glatzel, WLB)

Besser und sinnhafter kann wohl die Lebenswelt einer Bibliothek kaum anschaulich gemacht werden. In diesen Blättern ist bereits alles vorhanden, was Reicherts Werk ausmacht: ein Umfassen aller Kulturen und Epochen von der Antike bis zum 20. Jahrhundert, von Textfragmenten altgriechischer Dichtung über Bibeltexte, orientalische Dichtung bis hin zu Goethe, Trakl und Kafka. Der Künstler war leidenschaftlicher Leser, ein pictor doctus, der die Dichtung fremder Kulturen auch in deren 
Lettern druckte: hebräisch, griechisch, arabisch, kyrillisch. Teilweise zeichnete und schnitt er diese - besonders die großen Typen - mit eigener Hand. Kein Wunder, dass seine wortbezogenen Werke vielfach in Bibliotheken, Universitäten, Gerichten bis hin zur Schlosskapelle Mochental präsent sind. Das Wertvollste, was sein Lehrer HAP Grieshaber seinem Schüler vermittelte, war vielleicht der Auftrag für die Marbacher Expressionisten-Ausstellung 1960, der eigentlich inm zugedacht war. Durch diese noble Geste und praktische Unterstützung bei der Realisierung dieser ersten Blätter in Riesenformaten gab Grieshaber dem jungen Mann Sichtbarkeit, die später zum Auftrag für die Stuttgarter Drucke führte, der zweite wichtige Schritt für den Künstler. Ein Unverwechselbarer werkte da in unruhigen Zeiten, in denen künstlerische Richtungen und Moden rasch einander ablösten. Wo diverse Kunstrichtungen und Tendenzen weltweite Herrschaft antraten - etwa Op Art oder Pop Art - machte einer etwas, was sonst keiner machte: Bilder aus einem einzigartigen Material, architektonisch gebaute, in jeglichem Format als monumental zu bezeichnende Textblätter.

Den von Grieshaber übernommenen Begriff der „Poesia typographica“ hat er für eine Werkgruppe seiner Blätter übernommen und dazu sehr erhellend Folgendes formuliert:

\section{„poesia typographica”}

es gibt ein kleines buch von grieshaber, das er so genannt hat. Ich habe diesen begriff - wie auch das werkman'sche hot printing - ohne bedenken für meine arbeit in anspruch genommen; mögen mir beide verzeihen.

die poesia typographica ist lyrisch, architektonisch, auch geometrisch und musikalisch, trotzdem ist sie immer sie selber.

produkt einer druckindustrie, eines typo- oder designstudios oder gar eines kunstgewerbes ist sie nicht.

typografie ohne sprache

ohne worte

ohne buchstaben

typografie als bild typografie mit buchstaben

mit worten

mit linien

mit punkten

typografie mit unlesbaren worten

mit buchstaben aus farbe

poesie, die durch typografie entsteht

poesie, die durch drucken entsteht.

Diese fruchtbare Spannung, dieses Wechselspiel von textloser Typographie und typographisch monumentalisiertem Text ist wohl das entscheidende Charakteristikum in Reicherts Werk. Er ist ein Maler ohne Pinsel, der Meister-Typograph ohne Ausbildung, der ein formidabler, sensibler Drucker wurde. Oft, wie bei den ersten Blättern, entstehen seine Drucke in den großen Formaten ohne Maschine, sondern als sogenannte Reiberdrucke, buchstäblich „mit Löffel und Sandale“, wie es der Titel einer Ausstellung mit Blättern von Reichert formulierte. Dadurch ist jeder dieser Drucke ein Einzelwesen, keiner wie der andere - nicht als Zufallsergebnis, sondern im Sinne planvollen Durchspielens von Möglichkeiten und der Fähigkeit, den "Zufall" zum konstitutiven Gestaltungselement werden zu lassen.

Das ganze Werk Reicherts besteht aus Hochdruck, steht also in der Gutenbergtradition. Sein Wahlspruch war "printing is a way of life". Gelegentlicher ergänzender Einsatz von Offset und Siebdruck wurde von befreundeten Kollegen übernommen. Die Typen erwarb er soweit möglich, ansonsten entwarf und schnitt er sie selbst, wie etwa die Ginkgo-Blätter zu den Drucken mit Goethes Suleika-Gedichten. Dass diese ihm besonders am Herzen lagen, verwundert nicht, ist doch der „West-Östliche Diwan" das bedeutendste Werk, in dem sich Orient und Okzident nicht nur begegnen, sondern amalgamieren. Ein solcher Magier kultureller Verschmelzung war Reichert mit vielen Werken, so den Blättern des Zyklus "Schriftfest in Sofia", denen zu orientalischer Dichtung, sowie seinem opus magnum, dem "Haidholzer Psalter". Sein Werk wird, neben der Konstruktion von Buchstaben und Worten, sehr durch Farbigkeit bestimmt. Da gibt es die strengen, klassischen Druckfarben, aber im Zeitverlauf fortschreitend eine subtile Auffächerung mit selbst angemischten 
helleren Farben, in denen sowohl Einflüsse des Orients wie des von inm so geliebten Italien und besonders der Florentiner Manieristen spürbar werden.

Abb. 3: Schin (Arabischer Buchstabe Schin aus: Pietro della Valle: Reisebeschreibungen in Persien und Indien. Maximilian-Ges., 1981)

Aber diese vielfältigen, durchaus untereinander nicht kongruenten Einflüsse bedeuten keine Unentschiedenheit oder Ambivalenz. Ganz im Gegenteil besteht die Singularität seiner Drucke gerade darin, dass er aus all dem einen unverwechselbaren eigenen Stil schuf, ein dichtes idiosynkratisches Gewebe aus Anregungen und Selbsterdachtem. Anpassung wäre das letzte, was man bei diesem „bärtigen Widerborst" erwarten durfte.

Die Arbeiten Josua Reicherts sind streng gestaltet und zugleich gerade auch wegen der Farben voller Sinnlichkeit. Der lebensvolle Erzähler Reichert hat es einmal ganz einfach und lebensprall so formuliert: „... es ist schön, wenn sich neben der Presse die Holzlettern zu Türmen aufbauen und die Farbwalzen auf dem Drucktisch glänzen".

Wer je mit dem Drucken zu tun hatte, vergisst nie den charakteristischen Geruch einer Druckerwerkstatt. Das war auch bei Josua Reichert so. Die Werkstatt war kein Genieschuppen, sondern ein sorgfältig geordneter Raum, so wohlgeordnet wie seine Kunst. Zugleich war sie ein Archiv, in dem er praktisch sein gesamtes Werk in Belegstücken versammelt hatte, wie es im umfassenden Katalogwerk von Pfäfflin/Maurice dargestellt ist. Es wäre zu wünschen, dass dieses Ensemble erhalten bleibt, sei es am Ort oder anderswo in einem musealen Zusammenhang als Erinnerung an den Meisterdrucker.

Neben der Bewunderung seiner Kunst bewahren Freunde die Erinnerung an einen eindrucksvollen Mann: großgewachsen, kraftvoll, kommunikativ und zugleich einzelgängerisch, nicht einfach im Umgang, aber zugleich von feiner Empfindsamkeit und Zurückhaltung. Viele werden gute Erinnerungen an Werkstattbesuche, Ausstellungseröffnungen und fröhliche Stunden mit dem Andenken an Josua Reichert verbinden, der von sich einmal sagte: "Mein Leben ist ein Kontinuum".

Auch sein Werk in weit über tausend Bilderfindungen und zehntausenden von Druckexemplaren ist ein solches staunenswertes Kontinuum, das ein großes Konzept und zugleich eine überwältigende Vielfalt umfasst.

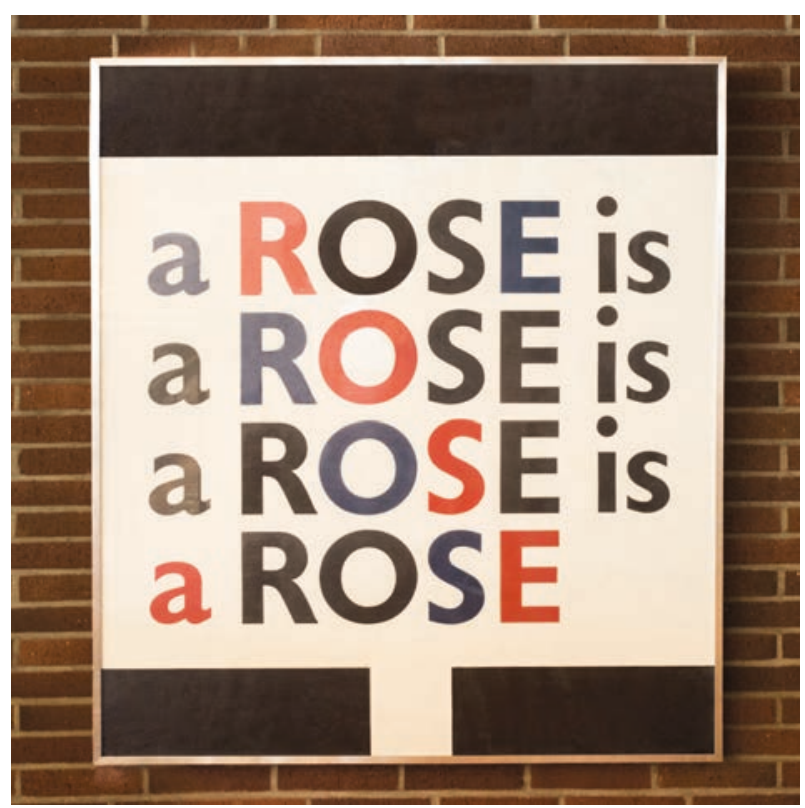

Abb. 4: Stuttgarter Drucke: a ROSE is a ROSE (Foto: Rafael Glatzel, WLB)

„Poesia typographica“ im erweiterten Sinn ist sein Vermächtnis: die Besucher der Landesbibliothek können sich daran immer neu erfreuen.

Wulf D. von Lucius 